\title{
The prognosis of invasive micropapillary carcinoma compared with invasive ductal carcinoma in the breast: a meta-analysis
}

Yun $\mathrm{Wu}^{1 \dagger}$, Ning Zhang ${ }^{1 \dagger}$ and Qifeng Yang ${ }^{1,2^{*}}$

\begin{abstract}
Background: Invasive micropapillary carcinoma (IMPC) of the breast is a rare variant of invasive ductal carcinoma (IDC). The prognosis of IMPC compared with that of IDC remains controversial; we conducted a meta-analysis to evaluate the prognostic difference between IMPC and IDC.

Methods: We searched the PubMed, Cochrane Library, and EMBASE databases for relevant studies comparing overall survival (OS), disease-specific survival (DSS), relapse-free survival (RFS), local-regional recurrence-free survival (LRRFS) or distant metastasis-free survival (DMFS) rates between IMPC and IDC. Fixed-effect and random-effect models were utilized based on the heterogeneity of the eligible studies. Heterogeneity was further evaluated by subgroup and sensitivity analyses.

Results: Fourteen studies with 1888 IMPC patients were included in the meta-analysis. The summarized odds ratio (OR) and $95 \%$ confidence interval $(95 \% \mathrm{Cl})$ was calculated to estimate the prognostic difference between IMPC and IDC. IMPC patients showed an unfavorable prognosis for RFS (OR; $2.04 ; 95 \% \mathrm{Cl}: 1.63-2.55)$ and LRRFS (OR: $2.82 ; 95 \%$ Cl: 1.90-4.17) compared with IDC. However, no significant difference was observed in OS (OR: 0.93; 95\% Cl: 0.78-1.10), DSS (OR: 1.16; 95\% Cl: 0.95-1.40) and DMFS (OR: 0.95; 95\% Cl: 0.67-1.35) between IMPC and IDC. No obvious statistical heterogeneity was detected, except for DSS. Funnel plots and Egger's tests did not reveal publication bias, except for RFS.

Conclusions: This analysis showed that IMPC patients have a higher rate of loco-regional recurrence than IDC patients. However, OS, DSS and DMFS were not significantly different between IMPC and IDC. These results could help clinicians select therapeutic and follow-up strategies for IMPC patients.
\end{abstract}

Keywords: Invasive micropapillary carcinoma, IMPC, Breast cancer, Prognosis, Survival, Meta-analysis

\section{Background}

Among females, breast cancer is the most common malignancy in both the developing and developed world, with over 1.3 million cases diagnosed and nearly 0.5 million deaths annually [1]. The 5-year survival rate of breast cancer was $89 \%$ from 2004 to 2010 in the United States, which significantly increased during the past 20 years due to early diagnosis through screening and the use of adjuvant systemic therapies [2,3]. Different breast cancer subtypes show various prognoses, since

\footnotetext{
* Correspondence: qifengy_sdu@163.com

${ }^{\dagger}$ Equal contributors

'Department of Breast Surgery, Qilu Hospital, Shandong University, No.107 West Wenhua Road, Jinan, Shandong 250012, People's Republic of China

${ }^{2}$ Pathology Tissue Bank, Qilu Hospital, Shandong University, No.107 West

Wenhua Road, Jinan, Shandong 250012, People's Republic of China
}

breast cancer is essentially a heterogeneous disease. Breast cancer heterogeneity can be detected at different levels, and morphological heterogeneity manifests as different histologic types [4].

According to the most recent World Health Organization (WHO) classification, breast cancer can be classified in up to 21 distinct histological types on the basis of cell morphology, growth, and architecture patterns [5]. The most common histological type is invasive carcinoma of no special type, also known as invasive ductal carcinoma (IDC), which accounts for approximately $75 \%$ of all invasive breast cancers [5]. However, invasive micropapillary carcinoma (IMPC) was listed as a rare subtype of invasive breast 
carcinoma and constitutes between $2 \%$ and $8 \%$ of all breast cancers [5-12].

Invasive micropapillary carcinoma (IMPC) was first described in breast cancer as having an "exfoliative appearance" by Fisher et al. [13] in 1980, and first proposed as the term of "invasive micropapillary carcinoma" by Siriaunkgul et al. [14] in 1993. In clinical presentations, IMPC patients typically show a palpable mass with no obvious difference from IDC in radiographic findings, locations, and gross features [15]. Therefore, the microscopic evaluation of histological differences is vital to clinical diagnosis.

Compared with IDC, IMPC shows a more distinct morphologic architecture, characterized by pseudopapillary and tubuloalveolar arrangements of tumor cell clusters in sponge-like, clear empty spaces, thereby mimicking extensive lymphatic invasion [14]. The neoplastic cell displayed an "inside-out" pattern, known as reverse polarity, which could be observed by electron microscopy. Immunohistochemistry (IHC) for epithelial membrane antigen and sialyl Lewis $\mathrm{X}$ was applied to highlight the apical cellular membrane of peripheral cells at tumor nests and to confirm the diagnosis [16].

According to clinical experience, most patients with IMPC present extensive axillary lymph node (ALN) metastasis at initial diagnosis. Several studies of this subtype of breast cancer have demonstrated that IMPC has high proclivity to lymphovascular invasion (LVI) (35\%-75\%), ALN metastasis (44\%-85\%), local recurrence and distance metastasis $[8,10,17,18]$, which has gained increasing attention in the past 30 years. Due to highly lymphotropic nature and more aggressive behavior of IMPC, it was generally accepted that this disease shows unfavorable prognosis compared with IDC $[10,17]$. Nevertheless, a number of recent studies have indicated that IMPC exhibited better or similar prognosis compared with IDC [19, 20]. For instance, Chen et al. [20] and Yu et al. [21] found that the overall survival (OS) was similar for IMPC and IDC patients; Chen et al. [20] also found that IMPC patients show a more favorable disease-specific survival (DSS) compared with IDC patients. However, Shi et al. [22] and Chen et al. [17] found that OS and relapse-free survival (RFS) were worse in the IMPC group than in the IDC group.

Because of the low incidence of this breast cancer variant and the relative scarce publications, there is some controversy about the prognosis of IMPC compared with IDC. The aim of current meta-analysis was to analyze the existing data to gain a clearer insight into the prognostic difference between IMPC and IDC. The results could help clinicians to determine counseling and follow-up strategies as well as tailor therapies to improve the outcomes of IMPC patients.

\section{Methods}

\section{Publication selection}

We searched PubMed and EMBASE databases and the Cochrane library in May 2017 for all studies reporting on IMPC and prognosis. Publications with the following search words in the title, abstract or key words were included: breast cancer, invasive carcinoma, micropapillary, invasive micropapillary carcinoma, prognosis, outcome and survival. Only studies written in English were included.

\section{Inclusion and exclusion criteria}

The articles were included in the present analysis if they met the following criteria: (I) studies involved must compare the prognostic outcomes between IMPC and IDC; (II) detailed statistics should report outcomes, such as percentages of overall survival (OS), disease-specific survival (DSS), relapse-free survival (RFS), local-regional recurrence-free survival (LRRFS) or distant metastasis-free survival (DMFS); and (III) if the studied population was duplicated, only the previous publication or the publication with the largest sample size would be included. Publications meeting all three criteria would be included; otherwise, studies were excluded from this analysis to avoid the significant heterogeneity between studies and bias of this analysis. Reviews, meeting abstracts, letters, comments, editorials, and case reports were also excluded because of the limited data.

\section{Patterns of failure and survival analysis}

We defined loco-regional recurrence (LRR) as the appearance of tumors in the ipsilateral breast, chest wall, ipsilateral axilla, internal mammary, supraclavicular area or infraclavicular area. Otherwise, recurrence was categorized as distant metastasis.

Overall survival (OS) and disease-specific survival (DSS) were measured from the data of surgery or diagnosis to the death and death due to breast cancer progression. Recurrence-free survival (RFS), LRR-free survival (LRRFS) and distant metastasis-free survival (DMFS) were measured from the date of operation to the date of LRR and/or distant metastasis, LRR and distant metastasis, respectively.

\section{Data extraction}

The following information was extracted from each eligible publication: author names, year of publication, total number of IMPC and IDC patients, components of IMPC, TNM stage, duration of follow-up and survival data (percentages and number of events). When the essential information was not provided in the articles, every effort was made to contact the authors. 


\section{Statistical analysis}

The summarized statistics of odd ratios (ORs) and 95\% confidence intervals (CIs) were calculated using a fixedeffect model when there was minimal heterogeneity among studies to assess the correlation between IMPC and prognosis. The $\chi^{2}$ and $\mathrm{I}^{2}$ test methods were utilized to determine the heterogeneity across studies of the ORs. When $p<0.05$ and/or $\mathrm{I}^{2}>50 \%$ which indicate significant heterogeneity, the random-effect model was used. Reasons for statistical heterogeneity were explored through subgroup or sensitivity analyses (when $\mathrm{I}^{2}>$ $50 \%)$. Each publication was weighted according to the size of the sample. Forest plots were generated using standard techniques to pool the included studies, for which horizontal lines signify $95 \% \mathrm{CI}$, the area of each square represents the weight, and the position of each square demonstrates the OR estimate. The vertical line at the null value showed that OR was 1.0. The overall summarized prognostic estimate under fixed effects with its CI was shown.

The potential publication bias was evaluated by the funnel plot and Egger's tests. An asymmetrical plot suggests potential publication bias. Funnel plot asymmetry was assessed by Egger's linear regression test, which was applied to measure funnel plot asymmetry on the natural logarithm scale of the OR. The significance of the intercept was determined by the t-test according to Egger $(p<0.05$ was considered representative of statistically significant publication bias). All $p$ values were twosided, and $p<0.05$ was considered as statistically significant. All statistical analyses were performed with STATA software (version 12.0; Stata Corporation, College Station, TX, USA).

\section{Results}

\section{Eligible studies}

The search yielded 642 publications. After removing the duplicates, the titles of the remaining publications were evaluated, and 299 publications were initially excluded. The abstracts of the publications 100 records were screened, and 61 publications were excluded. Thirtynine publications were potential eligible studies that mentioned identifying the correlation of IMPC and prognosis. Twenty-five studies were excluded for not providing adequate, detailed statistics or not comparing with IDC. Thus, based on the criteria described above, 14 publications were eligible for inclusion in this metaanalysis $[12,17,19-30]$. The search strategy and filters applied are shown in Fig. 1.

\section{Study characteristics}

Ultimately, a total of 1888 IMPC patients from the 14 publications, which had survival data, were included in this meta-analysis. The characteristics of these studies are detailed in Table 1 . Most $(n=13)$ of the studies recruited patients with all subtype IDC patients as the control group, but one study focused on the triple negative IDC [24]. In clinical practice, the term "pure IMPC" is defined as IDC constituting at least $75 \%$ of the micropapillary component [16]. The patients in two studies were pure IMPC patients, the patients in eleven studies were mixed patients, and the patients in one study were unknown.

\section{Survival outcomes of IMPC and IDC}

Only 8 of the 14 studies provided OS data $(n=301,041$ patients). The ORs and 95\% CIs for each study and the summarized OR are shown in Fig. 2a. The individual OR of the 8 articles ranged from 0.51 to 2.33 . The overall summarized estimate OR was 0.90 (95\% CI: 0.76-1.06). There was no significant heterogeneity across the studies $\left(\mathrm{I}^{2}=46.8 \%, \mathrm{x}^{2}=13.15, p=0.068\right)$. Using the randomeffects method yielded a similar effect estimate $(\mathrm{OR}=$ 0.94, 95\% CI: 0.67-1.32).

Seven studies ( $n=300,528$ patients) provided DSS data. The ORs and 95\% CIs for each study and the summarized OR are shown in Fig. 2b. The OR from each of the 6 studies ranged from 0.69 to 2.69 . The overall summarized estimate OR was 1.16 (95\% CI: 0.95-1.40), with a higher heterogeneity $\left(\mathrm{I}^{2}=84.5 \%, \mathrm{X}^{2}=38.63, p<0.001\right)$.

Nine articles $(n=4259$ cases $)$ provided RFS data. The OR and $95 \%$ CI for each study and the summarized OR are shown in Fig. 3a. The ORs of the 8 studies ranged from 0.67 to 2.68 . The overall summary estimate OR was 2.04 (95\% CI: $1.67-2.50)$, with no significant evidence of heterogeneity $\left(\mathrm{I}^{2}=20.8 \%, \mathrm{X}^{2}=10.10, p=0.27\right)$.

Five studies $(n=2323$ patients) provided LRRFS data. The OR and $95 \% \mathrm{CI}$ for each study and the summarized OR are shown in Fig. 3b. The OR from each of the 4 studies ranged from 1.99 to 5.01 . The overall summarized estimate OR was 3.24 (95\% CI: 2.32-4.53). Heterogeneity testing revealed $\mathrm{I}^{2}=3.8 \%$ and $\mathrm{X}^{2}=4.16, p=0.39$.

Five studies $(\mathrm{n}=2323$ patients) provided DMFS data. The OR and 95\% CI for each study and the summary OR are shown in Fig. 3c. The OR from each of the 4 studies ranged from 0.69 to 1.30 . The overall summarized estimate OR was 0.87 (95\% CI: 0.64-1.17). There was also no significant heterogeneity across the studies $\left(\mathrm{I}^{2}=0.0 \%, \mathrm{X}^{2}=3.18, p=0.53\right)$.

\section{Publication bias}

Funnel plots were generated to detect potential publication bias (Additional file 1: Figure S1 and Additional file 2: Figure S2), which did not reveal remarkable asymmetry. Egger's tests were also performed to detect publication bias to diminish the subjective bias. No evident publication bias was detected by Egger's tests for survival data, except for RFS. The $p$-values for Egger's test of OS, DSS, 


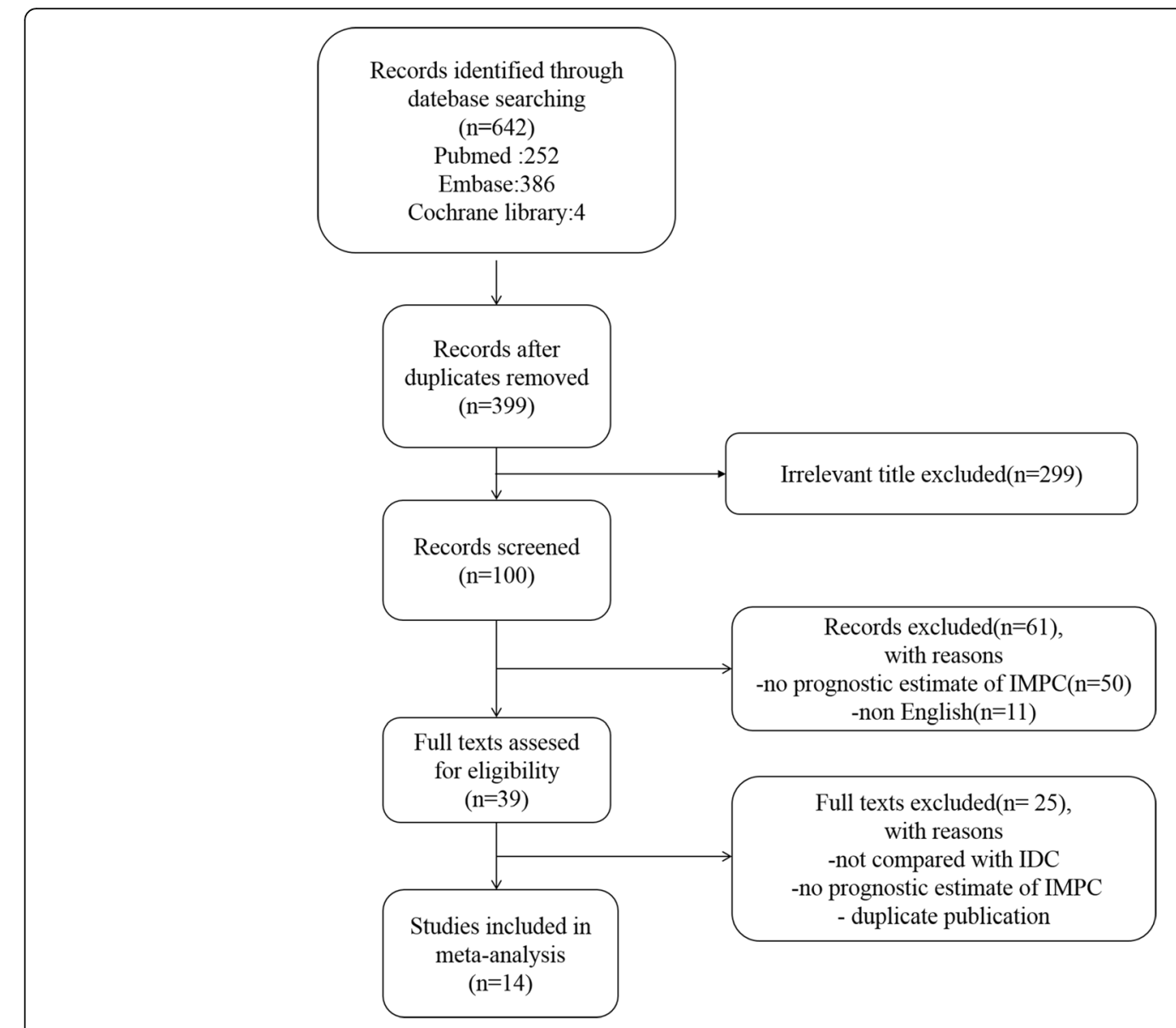

Fig. 1 Flow diagram illustrating the selection of the included studies

RFS, LRRFS and DMFS were 0.89, 0.44, 0.04, 0.271 and 0.83 , respectively.

\section{Discussion}

Breast cancer is the most common cancer in women and the primary cause of death among women globally [2]. Breast cancer heterogeneity can be detected at different levels, from the classic histopathological characterization to the more modern molecular classification. According to the WHO, breast cancer can be classified into 21 distinct histological types, which include non-invasive breast cancer, including ductal carcinoma in situ, lobular carcinoma in situ and Paget's disease and invasive breast cancers, such as invasive ductal carcinoma and some rare cancer types [5].

IMPC of the breast is a morphologically distinct subtype of breast carcinoma, associated with a highly lymphotropic nature and aggressive clinicopathological features and tends to have a higher clinical stage at initial diagnosis in IMPC patients [20]. IDC is the most common type of breast cancer, accounting for up to $75 \%$ of invasive breast carcinomas [15]. In clinical practice, the histopathological classification has a prognostic value. Clinicians typically presumed that a more histopathologically aggressive carcinoma may indicate a worse prognosis. However, there remains discordance in the published literatures regarding whether IMPC patients show a worse prognostic outcome than IDC patients.

Numerous reports have been published in the past three decades to compare the prognostic difference between IMPC and IDC [12, 17, 19-30]. Chen et al. [20], using the US National Cancer Institute's Surveillance, Epidemiology, and End Results (SEER) database, found that the overall prognostic results of IMPC were similar to those of IDC and showed a more favorable DSS than IDC patients, although lymph node metastasis was more common in the former. Yu et al. [21] demonstrated a significantly worse LRRFS and RFS for IMPC compared with matched IDC controls, however no difference was observed in the overall survival analysis. In contrast, Shi et al. [22] and Chen et al. [17] showed that OS and RFS were poorer in the IMPC group than in the IDC group. However, since the incidence of IMPC was low, the majority of studies only reported on small sample sizes with limited follow-ups. The present meta-analysis 
Table 1 Characteristics of eligible studies

\begin{tabular}{|c|c|c|c|c|c|c|c|c|c|c|c|}
\hline \multirow[t]{2}{*}{ Study } & \multirow{2}{*}{$\begin{array}{l}\text { Study } \\
\text { period }\end{array}$} & \multicolumn{2}{|c|}{ No. of cases } & \multirow{2}{*}{$\begin{array}{l}\text { Component } \\
\text { of IMPC* }\end{array}$} & \multirow{2}{*}{$\begin{array}{l}\text { TNM } \\
\text { stage }\end{array}$} & \multirow{2}{*}{$\begin{array}{l}\text { Follow-up } \\
\text { time(M) }\end{array}$} & \multicolumn{5}{|c|}{ Survival data ${ }^{a}$} \\
\hline & & IMPC & IDC & & & & OS & DSS & RFS & LRRFS & DMFS \\
\hline Tang [30] & 2017 & 170 & 728 & mixed & $||,,||||$, & median 40 & $\begin{array}{l}94.5 \% \text { vs } \\
90.6 \% \\
(P=0.592)\end{array}$ & - & $\begin{array}{l}82.1 \% \text { vs } \\
90.6 \% \\
(P=0.001)\end{array}$ & $\begin{array}{l}86.9 \% \text { vs } \\
97.1 \% \\
(P<0.001)\end{array}$ & $\begin{array}{l}91.5 \% \text { vs } \\
88.3 \% \\
(P=0.923)\end{array}$ \\
\hline Li [23] & 2016 & 33 & 347 & mixed & $||,,||||$, & median 39 & $\begin{array}{l}97 \% \text { vs } \\
94.2 \% \\
(P=0.78)\end{array}$ & - & $\begin{array}{l}87.9 \% \text { vs } \\
86.2 \% \\
(P=0.88)\end{array}$ & $\begin{array}{l}93.9 \% \text { vs } \\
89.0 \% \\
(P=0.88)\end{array}$ & $\begin{array}{l}90.9 \% \text { vs } \\
89.0 \% \\
(P=0.97)\end{array}$ \\
\hline Yu [21] & 2015 & 267 & 267 & mixed & $||,,||||$, & median 59 & $\begin{array}{l}97.7 \% \text { vs } \\
95.7 \% \\
(P=0.67)\end{array}$ & - & $\begin{array}{l}85.0 \% \text { vs } \\
92.1 \% \\
(P=0.07)\end{array}$ & $\begin{array}{l}91.8 \% \text { vs } \\
96.3 \% \\
(P=0.03)\end{array}$ & $\begin{array}{l}91.4 \% \text { vs } \\
93.3 \% \\
(P=0.52)\end{array}$ \\
\hline Chen [24] & 2015 & 95 & 200 & mixed & $||,,||||$, & median 60 & $\begin{array}{l}81.9 \% \text { vs } \\
79.8 \% \\
(P=0.475)\end{array}$ & - & - & $\begin{array}{l}71.4 \% \text { vs } \\
89.8 \% \\
(P<0.001)\end{array}$ & $\begin{array}{l}79.8 \% \text { vs } \\
73.7 \% \\
(P=0.091)\end{array}$ \\
\hline Shi [22] & 2014 & 188 & 1289 & mixed & $||,,||||$, & median40.5 & - & $\begin{array}{l}67.1 \% \text { vs } \\
87.5 \% \\
(P<0.001)\end{array}$ & $\begin{array}{l}75.9 \% \text { vs } \\
89.5 \% \\
(P<0.001)\end{array}$ & - & - \\
\hline Liu [19] & 2014 & 51 & 102 & mixed & $||,,||||$, & median 51 & - & - & $\begin{array}{l}84.3 \% \text { vs } \\
78.4 \% \\
(P=0.606)\end{array}$ & - & - \\
\hline Chen [20] & 2014 & 636 & 297,735 & unknown & $||||,,|||,| \mathrm{V}$ & median 48 & $\begin{array}{l}82.9 \% \text { vs } \\
80.5 \% \\
(P=0.086)\end{array}$ & $\begin{array}{l}91.8 \% \text { vs } \\
88.6 \% \\
(P=0.086)\end{array}$ & - & - & - \\
\hline Vingiani [25] & 2013 & 49 & 13,487 & pure & $||,,||||$, & median 51 & $\begin{array}{l}89.8 \% \text { vs } \\
90.8 \% \\
(P=0.80)\end{array}$ & - & $\begin{array}{l}75.5 \% \text { vs } \\
79.6 \% \\
(P=0.48)\end{array}$ & - & - \\
\hline Gokce [12] & 2013 & 103 & 34 & mixed & $||,||||$, & mean 63.5 & - & $\begin{array}{l}75.9 \% \text { vs } \\
82.4 \% \\
(P=0.44)\end{array}$ & - & - & - \\
\hline Kuba [26] & 2011 & 10 & 162 & mixed & $||,,||||$, & median 72 & - & $\begin{array}{l}90 \% \text { vs } \\
94.4 \%\end{array}$ & $\begin{array}{l}90 \% \text { vs } \\
89.8 \%\end{array}$ & - & - \\
\hline Yu [27] & 2010 & 72 & 144 & mixed & $|| I,,|I|$ & median 45 & $\begin{array}{l}86.0 \% \text { vs } \\
87.7 \% \\
(P=0.18)\end{array}$ & - & $\begin{array}{l}68.2 \% \text { vs } \\
81.4 \% \\
(P=0.046)\end{array}$ & $\begin{array}{l}84.7 \% \text { vs } \\
94.4 \% \\
(P=0.0024)\end{array}$ & $\begin{array}{l}78.1 \% \text { vs } \\
79.3 \% \\
(P=0.87)\end{array}$ \\
\hline Kim [28] & 2010 & 61 & 221 & pure & $|| I,,|I|$ & mean 38.6 & - & - & $\begin{array}{l}86.9 \% \text { vs } \\
94.6 \% \\
(P=0.049)\end{array}$ & - & - \\
\hline Chen [17] & 2008 & 100 & 100 & mixed & $\mathrm{I}, \mathrm{I}, \mathrm{III}, \mathrm{IV}$ & mean 60.1 & $\begin{array}{l}59 \% \text { vs } \\
77 \% \\
(P=0.004)\end{array}$ & $\begin{array}{l}63.3 \% \text { vs } \\
81 \% \\
(P=0.005)\end{array}$ & - & - & - \\
\hline Zekioglu [29] & 2004 & 53 & 60 & mixed & $|| I,,|I|$ & mean 56.5 & - & $\begin{array}{l}72.2 \% \text { vs } \\
81.7 \% \\
\text { NS }\end{array}$ & - & - & - \\
\hline
\end{tabular}

Abbreviations: IMPC, Invasive micropapillary carcinoma; IDC, Invasive ductal carcinoma; OS, Overall survival; DSS, Disease-specific survival; RFS, Relapse-free Survival; LRRFS, Local-regional recurrence free survival; DMFS, Distant metastasis-free survival; NS, Not significant

${ }^{a}$ All percentage was for IMPC vs IDC\%

${ }^{*}$ At least $75 \%$ of the micropapillary component identified in an IDC to be defined as pure IMPC

summarized the current evidence of the prognostic difference between IMPC and IDC. Based on the combined data of 14 eligible published studies, we observed that IMPC is an unfavorable prognostic factor for RFS $(p<$ $0.001)$ and LRRFS $(\mathrm{p}<0.001)$, while no significant difference was detected in OS $(p=0.20)$, DSS $(p=0.14)$ and DMFS $(p=0.35)$ between IMPC and IDC.

Eight of the fourteen studies referred to the OS, and only Chen et al. [17] suggested that the OS of IMPC is comparable to that of IDC. Furthermore, we observed that IMPC showed unfavorable RFS and LRRFS due to its lymphotropic nature and aggressive behavior. However, we revealed a similar DMFS compared with IDC since the aggressive behavior of IMPC mainly embodied a higher rate of lymph node involvement rather than distant metastasis, in contrast to another aggressive subtype, triple negative IDC [24]. We suspected that the treatment therapy may explain the contradiction between unfavorable local relapse and better overall survival. According to the guidelines of the National 


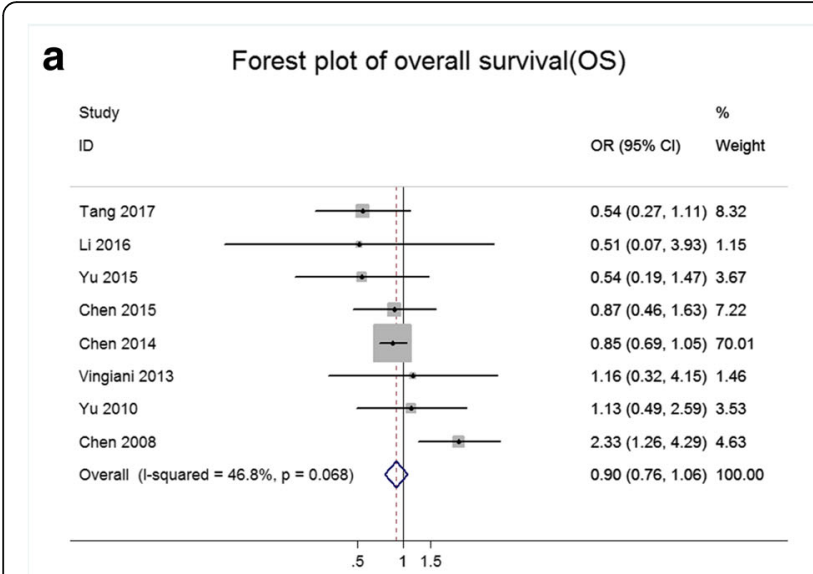

b Forest plot of disease-specific survival (DSS)

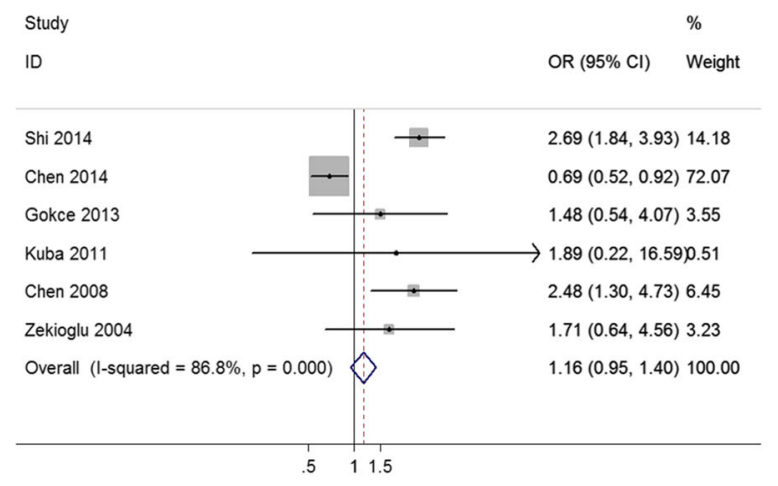

Fig. 2 Results of the survival analysis in IMPC compared with IDC. a Forest plot of the odds ratio (OR) for overall survival (OS) from eligible studies. b Forest plot of the odds ratio (OR) for disease-specific survival (DSS) from eligible studies

Comprehensive Cancer Network, the lymphatic involvement of breast cancer will always lead to radical surgical excision as well as post-mastectomy radiotherapy for patients with more than 3 involved nodes, which will contribute to the overall survival of these patients. Considering the higher rate of loco-regional recurrence in IMPC patients, we recommend that clinicians adopt sufficient local treatments, such as regional surgical resection and local radiotherapy. Moreover, clinicians should pay more attention to the loco-regional recurrence for IMPC patients at follow-up.

Two of the fourteen studies included pure IMPC patients, while eleven studies included mixed patients and the patients in one study were unknown. Most of the included patients are of mixed histological type. According to previous studies, IMPC may occur either alone or in combination with other histological types of breast cancer, such as IDC, mucinous carcinoma, and ductal carcinoma in situ [7, 24, 31], and most patients had mixed IMPC $[12,22]$. The prognosis might potentially be affected by other histological components. However, Chen et al. [17] showed that tumors containing less than a $25 \%$ IMPC components were still associated with a significantly higher incidence of LVI and LN metastasis than IDC. Ide et al. [11] observed that the presence of the IMPC component alone was a significant predictive factor for LN metastasis, even if it was detected in only a small proportion of the tumor. Marchio et al. [32, 33] demonstrated that in histological features and molecular genetic profiles, mixed IMPC patients were more closely related to pure IMPC patients than to IDC patients. Thus, we suspected that the presence of the IMPC component correlates with the aggressive behavior of the tumor, regardless of the IMPC component proportion.

Additionally, we did not observe any obvious statistical heterogeneity among the studies included in the present analysis, except for DSS. Thus, we performed subgroup and sensitivity analyses to explore the heterogeneity. Subgroup analysis revealed that race potentially caused heterogeneity (Additional file 3: Figure S3). We observed that Asian IMPC patients showed an unfavorable DSS compared with Asian IDC patients $(\mathrm{OR}=2.61,95 \% \mathrm{CI}=$ 1.88-3.61). However, IMPC was a favorable prognostic factor in Caucasian populations $(\mathrm{OR}=0.77,95 \% \mathrm{CI}=$ $0.60-0.99$ ). These results may be affected by different genetic backgrounds and socioeconomic factors that may influence a woman's adherence to recommendations for clinical breast examination and the likelihood of the woman to seek appropriate care when a breast mass is detected [34-36]. Thus, these factors may be related to the degree of lymph node positivity at initial diagnosis, which was a significant prognostic factor in IMPC patients. Chen et al. showed lower lymph node positivity $(52 \%)$ than the range $(68.8-90.5 \%)$ reported in other large studies of IMPC [6, 7, 17, 20, 27, 29, 37-39], likely accounting for the favorable prognosis of DSS in Caucasian populations. These results emphasize the importance of early diagnosis for IMPC patients. To evaluate the influence of a single study on the pooled OR, we performed a sensitivity analysis by estimating the average OR in the absence of each study. The results indicated that Chen et al. [20] influenced the OR of DSS. Chen et al. [20] concluded that IMPC showed a more favorable DSS than IDC, in contrast to the general understanding, thereby influencing the DSS heterogeneity. Chen et al. [20] speculated that higher ER positivity and a decreased rate of lymph node metastasis resulted in a favorable DSS outcome. Compared with other eligible studies, the most significant differences were TNM stage and the rate of lymph node positivity of IMPC patients, which may influence the eventual outcome. The lower lymph node positivity, lower TNM stage and notably slightly 
a Forest plot of relapse free survival(RFS)

Study

ID

Tang 2017

Li 2016

Yu 2015

Shi 2014

Liu 2014

Vingiani 2013

Kuba 2011

Yu 2010

Kim 2010

Overall (l-squared $=20.8 \%, p=0.258$ )

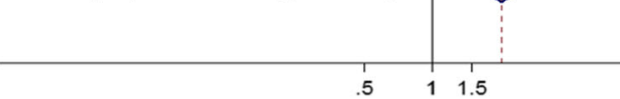

$\%$

Weight

b Forest plot of local-regional recurrence free survival(LRRFS)

Study

ID

Tang 2017

Li 2016

Yu 2015

Chen 2015

Yu 2010

Overall (I-squared $=3.8 \%, p=0.385$ )

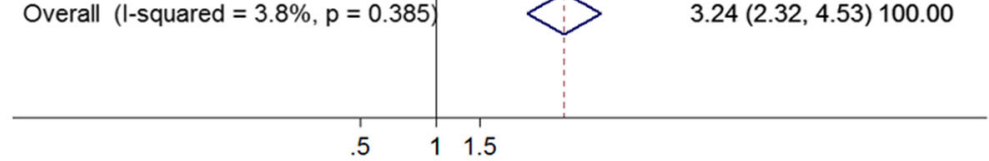

OR $(95 \% \mathrm{Cl}) \quad$ Weight
$5.00(2.68,9.34) 19.56$

$2.20(0.79,6.19) 10.83$

$1.99(0.94,4.21) 28.64$

$3.57(1.88,6.79) 26.06$

$3.53(1.50,8.32) 14.91$

$3.24(2.32,4.53) 100.00$

C Forest plot of distant metastasis-free survival(DMFS)

Study

ID

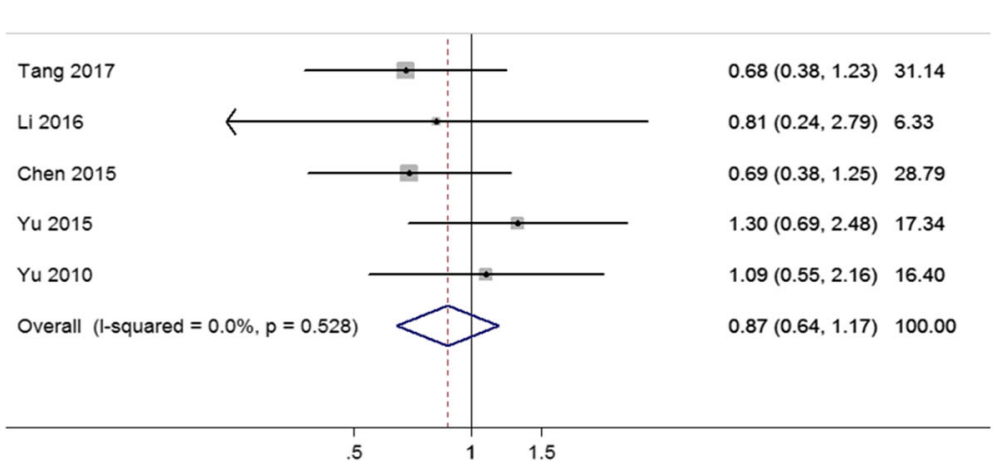

Fig. 3 (See legend on next page.) 
(See figure on previous page.)

Fig. 3 Results of the recurrence analysis in IMPC compared with IDC. a Forest plot of the odds ratio (OR) for relapse-free survival (RFS) from eligible studies. b Forest plot of the odds ratio (OR) for local-regional recurrence-free survival (LRRFS) from eligible studies. c Forest plot of the odds ratio (OR) for distant metastasis-free survival (DMFS) from eligible studies

higher mastectomy rate of IMPC patients from Chen et al. [20] may suggest that these patients received radical therapy in the initial stage of the disease, therefore showing a favorable DSS rate of IMPC patients.

Among all of the studies included in the present analysis, we did not detect significant statistical publication bias. However, Egger's test for RFS showed an obvious publication bias $(p=0.04)$, which may influence the robustness of the result of RFS. Thus, it cannot be determined whether the IMPC patients show a worse RFS than IDC patients. Notably, some studies that yielded negative results might not have been published at all, further increasing the potential influence of publication bias. The funnel plot generated to investigate this potential influence did not reveal any obvious asymmetry, and hence publication bias was not apparent.

Moreover, our meta-analysis has several limitations requiring consideration. First, the number of available, eligible publications is relatively small and may contribute to potential publication bias. Only scattered studies provided information concerning LRRFS and DMFS in the IMPC and IDC patients. Second, important pathological parameters, such as grade, molecular type (her2 status, hormone receptors), components of IMPC and administered therapies, such as chemotherapy, radiotherapy, etc., which may play a prognostic role and affect management decisions, were not consistently reported across all studies and was not considered in the present metaanalysis. Third, there were some notable clinical differences among the eligible studies (i.e., the criteria of eligible patients, component of IMPC, TNM stages, molecular subtypes of the patients included, median age and duration of follow-up) requiring further discussion. Finally, the retrospective design of the studies included in the present meta-analysis limits its power. Future prospective trials are needed to validate these results.

\section{Conclusions}

The present study is the first meta-analysis to explore the prognostic differences between IMPC and IDC. The results suggested that IMPC patients have a higher rate of loco-regional recurrence than IDC patients, although no obvious difference in OS, DSS and DMFS compared with IDC was detected. More large-scale retrospective studies or prospective clinical trials of IMPC are needed to contribute to individualized and tailored therapy, which may improve the clinical management and outcomes of IMPC.

\section{Additional files}

Additional file 1: Figure S1. (A) Funnel plot to detect publication bias for overall survival (OS). (B) Funnel plot to detect publication bias for disease-specific survival (DSS). (TIFF $304 \mathrm{~kb}$ )

Additional file 2: Figure S2. (A) Funnel plot to detect publication bias for relapse-free survival (RFS). (B) Funnel plot to detect publication bias for localregional recurrence-free survival (LRRFS). (C) Funnel plot to detect publication bias for distant metastasis-free survival (DMFS). (TIFF $261 \mathrm{~kb}$ )

Additional file 3: Figure S3. Subgroup analysis of DSS according to different ethnicities. (TIFF $1627 \mathrm{~kb}$ )

\section{Abbreviations}

95\% Cl: 95\% confidence interval; ALN: Axillary lymph node; DMFS: Distant metastasis-free survival; DSS: Disease-specific survival; IDC: Invasive ductal carcinoma; IMPC: Invasive micropapillary carcinoma; LRRFS: Local-regional recurrence free survival; LVI: Lymphovascular invasion; OR: Odds ratio; OS: Overall survival; RFS: Relapse-free Survival

\section{Acknowledgments}

The authors would like to thank Dr. Wenjing Zhao (PhD) for statistical assistance.

\section{Funding}

This study was funded by the National Natural Science Foundation of China (No.81672613), Key Research and Development Program of Shandong Province (No.2015GSF118093; No.2016GGE2775) and Shandong Science and Technology Development Plan (No.2016CYJS01A02) to Qifeng Yang and the National Natural Science Foundation of China (No. 81502285) to Ning Zhang. None of the funding bodies had any part in the design of the study and collection, analysis, and interpretation of data. The funding bodies supported the expense of the language editing of this manuscript.

Availability of data and materials

All data generated or analyzed during this study are included in this published article.

\section{Authors' contributions}

QY designed the study, YW and NZ collected the references, analyzed the data and drafted the manuscript, YW generated the figures and QY modified and approved the manuscript. All authors read and approved the final manuscript.

Ethics approval and consent to participate Not applicable.

Consent for publication Not applicable.

Competing interests

The authors declare that they have no competing interests.

\section{Publisher's Note}

Springer Nature remains neutral with regard to jurisdictional claims in published maps and institutional affiliations. 
Received: 24 May 2017 Accepted: 28 November 2017 Published online: 11 December 2017

\section{References}

1. Benson JR, Jatoi I. The global breast cancer burden. Future oncology (London, England). 2012;8:697-702.

2. Siegel RL, Miller KD, Jemal A. Cancer statistics, 2015. CA Cancer J Clin. 2015; 65:5-29.

3. Berry DA, Cronin KA, Plevritis SK, Fryback DG, Clarke L, Zelen M, Mandelblatt JS, Yakovlev AY, Habbema JD, Feuer EJ. Effect of screening and adjuvant therapy on mortality from breast cancer. N Engl J Med. 2005:353:1784-92.

4. Northridge ME, Rhoads GG, Wartenberg D, Koffman D. The importance of histologic type on breast cancer survival. J Clin Epidemiol. 1997;50:283-90.

5. Lakhani SR: Who classification of tumours of the breast. International Agency for Research on Cancer, 2012.

6. Paterakos M, Watkin WG, Edgerton SM, Moore DH 2nd, Thor AD. Invasive micropapillary carcinoma of the breast: a prognostic study. Hum Pathol. 1999:30:1459-63.

7. Nassar H, Wallis T, Andea A, Dey J, Adsay V, Visscher D. Clinicopathologic analysis of invasive micropapillary differentiation in breast carcinoma. Modern pathology : an official journal of the United States and Canadian Academy of Pathology, Inc. 2001;14:836-41.

8. Kuroda H, Sakamoto G, Ohnisi K, Itoyama S. Clinical and pathologic features of invasive micropapillary carcinoma. Breast cancer. 2004;11:169-74.

9. Pettinato G, Manivel CJ, Panico L, Sparano L, Petrella G. Invasive micropapillary carcinoma of the breast: Clinicopathologic study of 62 cases of a poorly recognized variant with highly aggressive behavior. Am J Clin Pathol. 2004;121:857-66.

10. De la Cruz C, Moriya T, Endoh M, Watanabe M, Takeyama J, Yang M, Oguma M, Sakamoto K, Suzuki T, Hirakawa H, Orita Y, Ohuchi N, Sasano H. Invasive micropapillary carcinoma of the breast: Clinicopathological and immunohistochemical study. Pathol Int. 2004:54:90-6.

11. Ide Y, Horii R, Osako T, Ogura K, Yoshida R, Iwase T, Akiyama F. Clinicopathological significance of invasive micropapillary carcinoma component in invasive breast carcinoma. Pathol Int. 2011:61:731-6.

12. Gokce H, Durak MG, Akin MM, Canda T, Balci P, Ellidokuz H, Demirkan B, Gorken IB, Sevinc Al, Kocdor MA, Saydam S, Harmancioglu O. Invasive micropapillary carcinoma of the breast: a clinicopathologic study of 103 cases of an unusual and highly aggressive variant of breast carcinoma. Breast J. 2013;19:374-81.

13. Fisher ER, Palekar AS, Redmond C, Barton B, Fisher B. Pathologic findings from the national surgical adjuvant breast project (protocol no. 4). Vi. Invasive papillary cancer. Am J Clin Pathol. 1980;73:313-22.

14. Siriaunkgul S, Tavassoli FA. Invasive micropapillary carcinoma of the breast Mod Pathol. 1993:6:660-2.

15. Gannon LM, Cotter MB, Quinn CM. The classification of invasive carcinoma of the breast. Expert Rev Anticancer Ther. 2013;13:941-54

16. Yang YL, Liu BB, Zhang X, Fu L. Invasive micropapillary carcinoma of the breast: an update. Archives of pathology \& laboratory medicine. 2016;140: 799-805.

17. Chen L, Fan Y, Lang RG, Guo XJ, Sun YL, Cui LF, Liu FF, Wei J, Zhang XM, Fu L. Breast carcinoma with micropapillary features: Clinicopathologic study and long-term follow-up of 100 cases. Int J Surg Pathol. 2008;16:155-63.

18. Luna-More S, de los Santos F, Breton JJ, Canadas MA. Estrogen and progesterone receptors, c-erbb-2, p53, and bcl-2 in thirty-three invasive micropapillary breast carcinomas. Pathol Res Pract. 1996;192:27-32.

19. Liu $Y$, Huang $X$, Bi R, Yang W, Shao Z. Similar prognoses for invasive micropapillary breast carcinoma and pure invasive ductal carcinoma: a retrospectively matched cohort study in china. PLoS One. 2014;9:e106564.

20. Chen AC, Paulino AC, Schwartz MR, Rodriguez AA, Bass BL, Chang JC, Teh BS. Population-based comparison of prognostic factors in invasive micropapillary and invasive ductal carcinoma of the breast. Br J Cancer. 2014:111:619-22

21. Yu JI, Choi DH, Huh SJ, Cho EY, Kim K, Chie EK, Ha SW, Park IA, Ahn SJ, Lee JS, Shin KH, Kwon Y, Kim YB, Suh CO, Koo JS, Kim JH, Jeong BG, Kim IA, Lee $J$ H, Park W. Differences in prognostic factors and failure patterns between invasive micropapillary carcinoma and carcinoma with micropapillary component versus invasive ductal carcinoma of the breast: retrospective multicenter case-control study (krog 13-06). Clinical breast cancer. 2015;15: 353-361.e352.
22. Shi WB, Yang LJ, Hu X, Zhou J, Zhang Q, Shao ZM. Clinico-pathological features and prognosis of invasive micropapillary carcinoma compared to invasive ductal carcinoma: a population-based study from china. PLoS One. 2014;9:e101390.

23. Li G, Yang S, Yao J, Wang Z, Yao G, Liu M, Ye C. Invasive micropapillary carcinoma of the breast had poor clinical characteristics but showed no difference in prognosis compared with invasive ductal carcinoma. World journal of surgical oncology. 2016;14:207.

24. Chen HL, Ding A. Comparison of invasive micropapillary and triple negative invasive ductal carcinoma of the breast. Breast. 2015;24:723-31.

25. Vingiani A, Maisonneuve P, Dell'orto P, Farante G, Rotmensz N, Lissidini G, Del Castillo A, Renne G, Luini A, Colleoni M, Viale G, Pruneri G. The clinical relevance of micropapillary carcinoma of the breast: a case-control study. Histopathology. 2013;63:217-24.

26. Kuba S, Ohtani H, Yamaguchi J, Hayashi H, Uga T, Kanematsu T, Shimokawa I. Incomplete inside-out growth pattern in invasive breast carcinoma: association with lymph vessel invasion and recurrence-free survival. Virchows Archiv : an international journal of pathology. 2011;458:159-69.

27. JI Y, Choi DH, Park W, Huh SJ, Cho EY, Lim YH, Ahn JS, Yang JH, Nam SJ. Differences in prognostic factors and patterns of failure between invasive micropapillary carcinoma and invasive ductal carcinoma of the breast: matched case-control study. Breast. 2010;19:231-7.

28. Kim SH, Hur SM, Lee SK, Kim WW, Kim S, Choe JH, Kim JH, Kim JS, Nam SJ, Yang JH, Lee JE. Characteristics of invasive micropapillary carcinoma of the breast: in comparison with invasive ductal carcinoma. J Breast Cancer. 2010; 13:174-9.

29. Zekioglu O, Erhan Y, Ciris M, Bayramoglu H, Ozdemir N. Invasive micropapillary carcinoma of the breast: high incidence of lymph node metastasis with extranodal extension and its immunohistochemical profile compared with invasive ductal carcinoma. Histopathology. 2004;44:18-23.

30. Tang SL, Yang JQ, Du ZG, Tan QW, Zhou YT, Zhang D, Lv Q. Clinicopathologic study of invasive micropapillary carcinoma of the breast. Oncotarget. 2017:8(26):42455-65.

31. Amendoeira I, Magalhaes J, Damasceno M. Invasive micropapillary carcinoma of the breast: are the pure forms more aggressive than the mixed forms? Breast J. 2003;9:337-8.

32. Marchiò C, Iravani M, Natrajan R, Lambros MBK, Geyer FC, Savage K, Parry S, Tamber N, Fenwick K, Mackay A, Schmitt FC, Bussolati G, Ellis I, Ashworth A, Sapino A, Reis-Filho JS. Mixed micropapillary-ductal carcinomas of the breast: a genomic and immunohistochemical analysis of morphologically distinct components. J Pathol. 2009;218:301-15.

33. Marchiò C, Iravani M, Natrajan R, Lambros MB, Savage $K$, Tamber N, Fenwick K, Mackay A, Senetta R, Di Palma S, Schmitt FC, Bussolati G, Ellis IO, Ashworth A, Sapino A, Reis-Filho JS. Genomic and immunophenotypical characterization of pure micropapillary carcinomas of the breast. J Pathol. 2008:215:398-410.

34. Svendsen RP, Paulsen MS, Larsen PV, Hansen BL, Stovring H, Jarbol DE, Sondergaard J. Associations between reporting of cancer alarm symptoms and socioeconomic and demographic determinants: a population-based, cross-sectional study. BMC Public Health. 2012;12:686.

35. Vernon SW, Vogel VG, Halabi S, Bondy ML. Factors associated with perceived risk of breast cancer among women attending a screening program. Breast Cancer Res Treat. 1993;28:137-44.

36. McDonald PA, Thorne DD, Pearson JC, Adams-Campbell LL. Perceptions and knowledge of breast cancer among african-american women residing in public housing. Ethnicity \& disease. 1999;9:81-93.

37. Pettinato G, Manivel CJ, Panico L, Sparano L, Petrella G. Invasive micropapillary carcinoma of the breast. Am J Clin Pathol. 2004;121:857-66.

38. Luna-More S, Casquero S, Perez-Mellado A, Rius F, Weill B, Gornemann I. Importance of estrogen receptors for the behavior of invasive micropapillary carcinoma of the breast. Review of 68 cases with follow-up of 54. Pathol Res Pract. 2000;196:35-9.

39. Walsh MM, Bleiweiss IJ. Invasive micropapillary carcinoma of the breast: eighty cases of an underrecognized entity. Hum Pathol. 2001;32:583-9. 